\title{
The Composition Operator and the Space of the Functions of Bounded Variation in Schramm-Korenblum's Sense
}

\author{
Z. Jesús, ${ }^{1}$ O. Mejía, ${ }^{1}$ N. Merentes, ${ }^{1}$ and S. Rivas ${ }^{2}$ \\ ${ }^{1}$ Departamento de Matemática, Universidad Central de Venezuela, Caracas 1220A, Venezuela \\ ${ }^{2}$ Instituto Universitario Tecnológico Elías Calixto Pompa, Guatire, Venezuela
}

Correspondence should be addressed to O. Mejía; odalism_18@yahoo.com

Received 20 March 2013; Accepted 20 July 2013

Academic Editor: Gen-Qi Xu

Copyright (C) 2013 Z. Jesús et al. This is an open access article distributed under the Creative Commons Attribution License, which permits unrestricted use, distribution, and reproduction in any medium, provided the original work is properly cited.

We show that the composition operator $H$, associated with $h:[a, b] \rightarrow \mathbb{R}$, maps the spaces $\operatorname{Lip}[a, b]$ on to the $\operatorname{space} \kappa \mathrm{BV}_{\phi}[a, b]$ of functions of bounded variation in Schramm-Korenblum's sense if and only if $h$ is locally Lipschitz. Also, verify that if the composition operator generated by $h:[a, b] \times \mathbb{R} \rightarrow \mathbb{R}$ maps this space into itself and is uniformly bounded, then regularization of $h$ is affine in the second variable.

\section{Introduction}

The composition operator problem (or COP, for short) refers to determining the conditions on a function $h: \mathbb{R} \rightarrow$ $\mathbb{R}$, such that the composition operator, associated with the function $h$, maps a space $\mathbb{X}$ of functions $u:[a, b] \rightarrow \mathbb{R}$ into itself $[1,2]$. There are several spaces where the COP has been resolved. For example, in 1961, Babaev [3] showed that the composition operator $H$, associated with the function $h: \mathbb{R} \rightarrow \mathbb{R}$, maps the space $\operatorname{Lip}[a, b]$ of the Lipschitz functions into itself if and only if $h$ is locally Lipschitz; in 1967, Mukhtarov [4] obtained the same result for the space $\operatorname{Lip}_{\alpha}[a, b]$ of the Hölder functions of order $\alpha(0<\alpha<1)$.

The first work on the COP in the space of functions of bounded variation $\mathrm{BV}[a, b]$ was made by Josephy in 1981 [5]. In 1986, Ciemnoczołowski and Orlicz [6] got the same result for the space of the functions of bounded $\varphi$ variation in Wiener's sense. In 1974, Chaika and Waterman [7] reached a similar result for the space of functions of bounded harmonic variation HBV $[a, b]$. In the years 1991 and 1995 Merentes showed a similar result for the spaces of absolutely continuous functions $\mathrm{AC}[a, b]$ and the space of function of bounded $\varphi$-variation in Riesz's sense $\operatorname{RV}_{\varphi}[a, b]$ (see $[8,9]$ ), and in 1998 Merentes and Rivas achieved the same result when the composition operator maps the space $\operatorname{RV}_{p}[a, b]$ of the functions of bounded $p$-variation in Riesz's sense $(1<p<\infty)$ into the space BV[a,b] [10]. In 2003, Pierce and Waterman solved the COP for the spaces $\phi \mathrm{BV}[a, b]$ and $\Lambda \mathrm{BV}[a, b]$ [11]. More recently, in 2011, Appell et al. [1] conclude the same results verifying when the composition operator maps $\operatorname{Lip}[a, b]$ into BV $[a, b]$. Finally, Appell and Merentes verify the same result for the space of functions of bounded $\kappa$-variation [12].

There exist spaces $\mathbb{X}$ of real functions defined on an interval $[a, b]$, such that $H$ maps $\mathbb{X}$ into itself and $h$ is not locally Lipschitz. For example, in the case the space of continuous functions $C[a, b]$ it follows from the TietzeUrysohn theorem that the composition operator $H$ acts from $C[a, b]$ into itself and the function $h$ must be continuous; that is, $h$ does not need to be Lipschitz. A similar result was obtained in the space of regulated functions [13].

A first objective of this work is to demonstrate that the composition operator, associated with the function $h$, maps the space $\operatorname{Lip}[a, b]$ of the Lipschitz functions into the space $\kappa \mathrm{BV}_{\phi}[a, b]$ of functions of bounded variation in SchrammKorenblum's sense or into the space $\kappa \mathrm{BV}[a, b]$ of functions of bounded variation in Korenblum's sense if and only if $h$ is locally Lipschitz. We also extend this result to function spaces $\mathbb{X}, \mathbb{Y}$, such that $\operatorname{Lip}[a, b] \subset \mathbb{X} \subset \mathbb{Y}$, where $\mathbb{Y} \subset \kappa \mathrm{BV}[a, b]$ or $\mathbb{Y} \subset \mathrm{BV}_{\phi}[a, b]$.

In a seminal article of 1982, Matkowski [14] showed that if the composition operator $H$, associated with the function $h$ : $[a, b] \times \mathbb{R} \rightarrow \mathbb{R}$, maps the space $\operatorname{Lip}[a, b]$ of the Lipschitzian 
functions into itself and is a globally Lipschitzian map, then the function $h$ has the form

$$
h(t, x)=\alpha(t) x+\beta(t), \quad t \in[a, b], x \in \mathbb{R},
$$

for some $\alpha, \beta \in \operatorname{Lip}[a, b]$.

There are a variety of spaces besides $\operatorname{Lip}[a, b]$ that verify this result [15]. The spaces of Banach $(\mathbb{X},\|\cdot\|)$ that fulfill this property are said to satisfy the Matkowski property [1].

In 1984, Matkowski and Miś [16] considered the same hypotheses on the operator $H$ for the space $\mathrm{BV}[a, b]$ of the function of bounded variation and concluded that (1) is true for the regularization $h^{-}$of the function $h$ with respect of the first variable; that is,

$$
h^{-}(t, x)=\alpha(t) x+\beta(t), \quad t \in[a, b], x \in \mathbb{R},
$$

where $\alpha, \beta \in \mathrm{BV}^{-}[a, b]$. Spaces that satisfy this conditions said to be verified Weak Matkowski Property [1].

A second objective of this paper is to show that if function $h(t, \cdot)$ is continuous in the second variable, for each $t \in[a, b]$, and the composition operator $H$, associated with the function $h$, is uniformly bounded, then $h$ satisfies (2).

\section{Preliminaries}

Let $[a, b]$ be a closed interval of the real line $\mathbb{R}(a, b \in \mathbb{R}, a<$ $b$ ). From now on, for a function $u:[a, b] \rightarrow \mathbb{R}$ denote by $L_{a}^{b}(u)$ the Lipschitz constant of $u$; that is,

$$
L_{a}^{b}(u)=\sup \left\{\frac{|u(t)-u(s)|}{|s-t|}: t, s \in[a, b], s \neq t\right\} .
$$

By $\operatorname{Lip}[a, b]=\left\{u:[a, b] \rightarrow \mathbb{R}: L_{a}^{b}(u)<\infty\right\}$ we will denote the space of the Lipschitz functions. It is well known that the space $\operatorname{Lip}[a, b]$ is a Banach space endowed with the norm

$$
\|u\|_{\operatorname{Lip}}:=|u(a)|+L_{a}^{b}(u) \quad(u \in \operatorname{Lip}[a, b]) .
$$

Ever since the notion of a function of bounded variation appeared, it has led to an incredible number of generalizations. In 1881, Jordan [17] introduced the definition of function of bounded variation for a function $u:[a, b] \rightarrow \mathbb{R}$ and showed that these kinds of function can be decomposed as the difference of two monotone functions. As a consequence of this result we have that those functions satisfy the Dirichlet criterion, that is, the functions that have pointwise convergent Fourier series.

Jordan defined such functions in the following way.

Definition 1. Let $u:[a, b] \rightarrow \mathbb{R}$ and $\pi: a \leq t_{1}<\cdots<t_{n} \leq b$ be a partition of the interval $[a, b]$. Consider

$$
\begin{gathered}
\sigma(u, \pi):=\sum_{j=1}^{n-1}\left|u\left(t_{j+1}\right)-u\left(t_{j}\right)\right|, \\
V(u ;[a, b])=\sup _{\pi} \sigma(u, \pi),
\end{gathered}
$$

where the supremum is taken over all partitions $\pi$ of the interval $[a, b]$.

If $V(u ;[a, b])<\infty$, then $u$ has bounded variation on the interval $[a, b]$ and this number is called the variation in Jordan's sense on $[a, b]$. This space of function is denoted by $\mathrm{BV}[a, b]$.

The concept of bounded variation has been the subject of intensive research, and many applications, generalizations, and improvements of them can be found in the literature (see for instance [18-20]). Some generalizations have been introduced by De La Vallée Poussin, F. Riesz, N. Wiener, L. C. Young, Yu. T. Medvedev, D. Waterman, B. Korenblum and M. Schramm.

In 1975, Korenblum in [21] considered a new kind of variation, called $\kappa$-variation, introducing a function $\kappa$ for distorting the expression $\left|t_{j}-t_{j-1}\right|$ in the partition itself rather than the expression $\left|f\left(t_{j}\right)-f\left(t_{j-1}\right)\right|$ in the range. Subsequently, this class of functions has been studied in detail by Cyphert and Kelingos [22]. One advantage of this alternate approach is that a function of bounded $\kappa$-variation may be decomposed into the difference of two simpler functions called $\kappa$-decreasing functions (for the precise definition see the following).

Definition 2. A function $\kappa:[0,1] \rightarrow[0,1]$ is said to be a $\kappa$-function or distortion function if it satisfies the following properties:

(1) $\kappa$ is continuous with $\kappa(0)=0$ and $\kappa(1)=1$,

(2) $\kappa$ is concave, increasing, and

(3) $\lim _{t \rightarrow 0^{+}}(\kappa(t) / t)=\infty$.

Simple examples of distortion functions are

$$
\kappa(t)=t^{\alpha}(0<\alpha<1), \quad \kappa(t)=t(1-\log t) .
$$

From Definition 2 we can see that $\kappa$ is subadditive; that is,

$$
\kappa(s+t) \leq \kappa(s)+\kappa(t) \quad(0 \leq s, t \leq 1),
$$

and since $\lim _{t \rightarrow 0^{+}}(\kappa(t) / t)=\infty$, then without loss of generality we can assume that

$$
\kappa(t) \geq t \quad(t \in[0,1]) .
$$

Furthermore Korenblum introduces the following concept of variation.

Definition 3. Let $\kappa:[0,1] \rightarrow[0,1]$ be a distortion function and $u:[a, b] \rightarrow \mathbb{R}$ and $\pi: a \leq t_{1}<\cdots<t_{n} \leq b$ a partition of the interval $[a, b]$. Consider

$$
\begin{gathered}
\kappa(u, \pi):=\frac{\sum_{j=1}^{n-1}\left|u\left(t_{j+1}\right)-u\left(t_{j}\right)\right|}{\sum_{j=1}^{n-1} \kappa\left(\left(t_{j+1}-t_{j}\right) /(b-a)\right)}, \\
\kappa V(u)=\kappa V(u ;[a, b]):=\sup _{\pi} \kappa(u, \pi),
\end{gathered}
$$

where the supremum is taken over all partitions $\pi$ of the interval $[a, b]$, called the $\kappa$-variation of $u$ on $[a, b]$. In the case, 
$\kappa V(u ;[a, b])<\infty$ we say that $u$ has $\kappa$-variation on $[a, b]$ and we will denote by $\kappa \mathrm{BV}[a, b]$ the space of functions of $\kappa$ variation on $[a, b]$.

In the case that $[a, b]=[0,1]$ the equality (9) becomes

$$
\kappa(u, \pi):=\frac{\sum_{j=1}^{n-1}\left|u\left(t_{j+1}\right)-u\left(t_{j}\right)\right|}{\sum_{j=1}^{n-1} \kappa\left(t_{j+1}-t_{j}\right)} .
$$

Some properties of the functions with bounded $\kappa$ variation are summarized in the following theorem.

Theorem 4 (see [22]). Let $\kappa:[0,1] \rightarrow[0,1]$ be a distortion function. Then

(1) $\kappa \mathrm{BV}[a, b]$ is a Banach space endowed with the norm

$$
\|u\|_{\kappa}=|u(a)|+\kappa V(u ;[a, b]) \quad(u \in \kappa \mathrm{BV}[a, b]) .
$$

(2) If the function $u$ is monotone, then $\kappa V(u ;[a, b])=$ $|u(b)-u(a)|$.

(3) If $u \in \kappa \mathrm{BV}[a, b]$, then $u$ is bounded and $\|u\|_{\infty} \leq$ $2\|u\|_{\kappa}$.

(4) $\operatorname{Lip}[a, b] \subset \operatorname{BV}[a, b] \subset \kappa \mathrm{BV}[a, b] \subset R[a, b]$, where $R[a, b]$ denotes the space of regulated functions.

(5) If $u \in \kappa \mathrm{BV}[a, b]$, then $u$ can be decomposed as difference of two $\kappa$-decreasing functions, that is, there exist functions $v:[a, b] \rightarrow \mathbb{R}$ such that $\sup _{s<t}((v(t)-$ $v(s)) / \kappa((t-s) /(b-a)))<\infty$.

It is easy to show that if $\lim _{t \rightarrow 0}(k(t) / t)$ is finite, then $\kappa \mathrm{BV}[a, b]=\mathrm{BV}[a, b]$. In [1] it is shown that inclusions (4) of Theorem 4 are strict.

Throughout this paper a $\varphi$-function $\varphi$ is a continuous increasing function $\varphi:[0, \infty) \rightarrow \mathbb{R}$, such that $\varphi(0)=0$ and $\lim _{t \rightarrow \infty} \varphi(t)=\infty$.

A $\phi$-sequence is a sequence of decreasing $\phi=\left\{\varphi_{n}\right\}_{n \geq 1}$ of convex $\varphi$-function that satisfies $\sum_{n \geq 1} \varphi_{n}(t)$ diverge for $t>0$.

We denote by $F_{\mathbb{N}}[a, b]$ the collection of finite or numerable family of nonoverlapping interval $\left\{\left[a_{n}, b_{n}\right]\right\}_{n \geq 1}$, such that $\bigcup_{n \geq 1}\left[a_{n}, b_{n}\right]=[a, b]$.

In 1985, Schramm [23] introduced a new concept of variation as follows.

Definition 5. Let $\phi=\left\{\varphi_{n}\right\}_{n \geq 1}$ be a $\phi$-sequence, $\left\{I_{n}=\left[a_{n}, b_{n}\right]\right\}_{n \geq 1} \in F_{\mathbb{N}}[a, b]$, and $u:[a, b] \rightarrow \mathbb{R}$. We define

$$
\begin{gathered}
\sigma_{\phi}\left(u, I_{n}\right):=\sum_{n=1}^{\infty} \varphi_{n}\left(\left|u\left(b_{n}\right)-u\left(a_{n}\right)\right|\right), \\
V_{\phi}(u)=V_{\phi}(u ;[a, b]):=\sup _{I_{n} \in F_{\mathbb{N}}[a, b]} \sigma_{\phi}\left(u, I_{n}\right) .
\end{gathered}
$$

If $V_{\phi}(u ;[a, b])<\infty$, we say that $u$ has bounded $\phi$-variation in the interval $[a, b]$ and this number denotes the $\phi$-variation of $u$ in Schramm's sense in $[a, b]$. The class of functions that have bounded $\phi$-variation in the interval $[a, b]$ is denoted by $V_{\phi}[a, b]$. The vectorial space generated by this class is denoted by $\mathrm{BV}_{\phi}[a, b]$.
The next lemma is useful for building the space generated by several classes of functions.

Lemma 6. Let $\mathbb{X}$ be a vector space and $A \subset \mathbb{X}$ a nonempty and symmetric set. Then

(1) $0 \in A$.

(2) The vector space generated for $A$ is equal to

$$
\langle A\rangle=\{x \in \mathbb{X}: \exists \lambda>0 \text { such that } \lambda x \in A\}=\bigcup_{\lambda>0} \lambda A .
$$

Some properties of functions of bounded $\phi$-variation in Schramm's sense are given in the following theorem.

Theorem 7 (see [23]). Let $\phi=\left\{\varphi_{n}\right\}_{n \geq 1}$ be $\phi$-sequence then

(1) $\mathrm{BV}_{\phi}[a, b]$ is a Banach space endowed with the norm

$$
\|u\|_{\phi}:=|u(a)|+\mu(u) \quad\left(u \in \mathrm{BV}_{\phi}[a, b]\right),
$$

$$
\text { where } \mu(u):=\inf _{\lambda>0}\left\{\lambda>0: V_{\phi}(u / \lambda) \leq 1\right\} .
$$

(2) If $u$ is monotone, then $V_{\phi}(u)=\varphi_{1}(|u(b)-u(a)|)$.

(3) If $u \in V_{\phi}[a, b]$, then $u$ is bounded and $\|u\|_{\infty} \leq|u(a)|+$ $2 \varphi_{1}^{-1}\left(V_{\phi}(u)\right)$.

(4) $V_{\phi}[a, b]$ is a symmetrical and convex set.

(5) $\mathrm{BV}_{\phi}[a, b]=\{u:[a, b] \rightarrow \mathbb{R}: \exists \lambda>0$ such that $\left.V_{\phi}(\lambda u)<\infty\right\}$.

(6) $u \in V_{\phi}[a, b]$, then $u$ has lateral limits at each point of $[a, b]$.

In 1986, S. K. Kim and J. Kim [24] combined the concepts of $\kappa$-variation and $\phi$-variation introduced by Korenblum and Schramm to create the concept of $\kappa \phi$-variation or variation in Schramm-Korenblum's sense.

Definition 8. Let $\kappa:[0,1] \rightarrow[0,1]$ be a distortion function, $\phi=\left\{\varphi_{n}\right\}_{n \geq 1}$ a $\phi$-sequence, $\left\{I_{n}=\left[a_{n}, b_{n}\right]\right\}_{n \geq 1} \in F_{\mathbb{N}}[a, b]$, and $u:[a, b] \rightarrow \mathbb{R}$. We define

$$
\begin{gathered}
\kappa \sigma_{\phi}\left(u, I_{n}\right):=\frac{\sum_{n=1}^{\infty} \varphi_{n}\left(\left|u\left(b_{n}\right)-u\left(a_{n}\right)\right|\right)}{\sum_{n=1}^{\infty} \kappa\left(\left(b_{n}-a_{n}\right) /(b-a)\right)}, \\
\kappa V_{\phi}(u)=\kappa V_{\phi}(u ;[a, b]):=\sup _{I_{n} \in F_{\mathbb{N}}[a, b]} \kappa \sigma_{\phi}\left(u, I_{n}\right) .
\end{gathered}
$$

If $\kappa V_{\phi}(u ;[a, b])<\infty$, we say that $u$ has bounded $\kappa \phi$ variation in the interval $[a, b]$ and this number denotes the $\kappa \phi$-variation of $u$ in Schramm-Korenblum's sense in $[a, b]$. The class of functions that have bounded $\kappa \phi$-variation in the interval $[a, b]$ is denoted by $\kappa V_{\phi}[a, b]$. The vectorial space generated by this class is denoted by $\kappa \mathrm{BV}_{\phi}[a, b]$.

A particular case of $\phi$-sequence is when all the functions $\varphi_{n}, n \in \mathbb{N}$ are equal to a fixed $\varphi$-function $\varphi$. In this situation the class $\kappa V_{\varphi}[a, b]$ is the class of the functions that have bounded $\kappa \varphi$-variation in Wiener-Korenblum's sense. This class of functions is denoted by $\kappa V_{\varphi}[a, b]$ and the vectorial 
space generated by this class of function is denoted by $\kappa \mathrm{BV}_{\varphi}[a, b]$.

Some properties of functions of bounded $\kappa \phi$-variation in Schramm-Korenblum's sense are given in the following theorem.

Theorem 9. Let $\kappa:[0,1] \rightarrow[0,1]$ be a distortion function and $\phi=\left\{\varphi_{n}\right\}_{n \geq 1}$ a $\phi$-sequence, then

(1) $\kappa \mathrm{BV}_{\phi}[a, b]$ is a Banach space endowed with the norm

$$
\|u\|_{\kappa \phi}:=|u(a)|+\mu_{\phi}(u) \quad\left(u \in \kappa \mathrm{BV}_{\phi}[a, b]\right)
$$

where $\mu_{\phi}(u):=\inf _{\lambda>0}\left\{\lambda>0: \kappa V_{\phi}(u / \lambda) \leq 1\right\}$.

(2) If $u$ is monotone, then $\kappa V_{\phi}(u)=\varphi_{1}(|u(b)-u(a)|)$.

(3) If $u \in \kappa V_{\phi}[a, b]$, then $u$ is bounded and $\|u\|_{\infty} \leq|u(a)|+$ $2 \varphi_{1}^{-1}\left(\kappa V_{\phi}(u)\right)$.

(4) $\kappa V_{\phi}[a, b]$ is a symmetrical and convex set.

(5) $\kappa \mathrm{BV}_{\phi}[a, b]=\{u:[a, b] \rightarrow \mathbb{R}: \exists \lambda>0$ such that $\left.\kappa V_{\phi}(\lambda u)<\infty\right\}$.

(6) $\mathrm{BV}[a, b] \subset V_{\phi}[a, b] \subset \kappa V_{\phi}[a, b]$, and therefore $\mathrm{BV}[a, b] \subset \mathrm{BV}_{\phi}[a, b] \subset \kappa \mathrm{BV}_{\phi}[a, b]$.

(7) $\kappa \mathrm{BV}[a, b] \subset \kappa \mathrm{BV}_{\phi}[a, b]$.

(8) $u \in \kappa V_{\phi}[a, b]$, then $u$ has lateral limits at each point of $[a, b]$.

Proof.

Part (1). See [24].

Part (2). We take $\left\{\left[a_{n}, b_{n}\right]\right\}_{n \geq 1} \in F_{\mathbb{N}}[a, b]$, then since the functions $0<t \rightarrow \varphi_{n}(t) / t$ are increasing, we obtain

$$
\begin{aligned}
& \frac{\sum_{n=1}^{\infty} \varphi_{n}\left(\left|u\left(b_{n}\right)-u\left(a_{n}\right)\right|\right)}{\sum_{n=1}^{\infty} \kappa\left(\left(b_{n}-a_{n}\right) /(b-a)\right)} \sum_{n=1}^{\infty} \frac{\varphi_{n}\left(\left|u\left(b_{n}\right)-u\left(a_{n}\right)\right|\right)}{\left|u\left(b_{n}\right)-u\left(a_{n}\right)\right|}\left|u\left(b_{n}\right)-u\left(a_{n}\right)\right| \\
& \quad \times\left(\sum_{n=1}^{\infty} \kappa\left(\frac{b_{n}-a_{n}}{b-a}\right)\right)^{-1} \\
& \leq \sum_{n=1}^{\infty} \frac{\varphi_{n}(|u(b)-u(a)|)}{|u(b)-u(a)|}\left|u\left(b_{n}\right)-u\left(a_{n}\right)\right| \\
& \quad \times\left(\sum_{n=1}^{\infty} \kappa\left(\frac{b_{n}-a_{n}}{b-a}\right)\right)^{-1} \\
& \leq \frac{\varphi_{1}(|u(b)-u(a)|)}{|u(b)-u(a)|} \sum_{n=1}^{\infty}\left|u\left(b_{n}\right)-u\left(a_{n}\right)\right| \\
&=\varphi_{1}(|u(b)-u(a)|),
\end{aligned}
$$

from which is obtained

$$
\kappa V_{\phi}(u) \leq \varphi_{1}(|u(b)-u(a)|) .
$$

From the Definition of $k V_{\phi}(u)$ we have the reciprocal inequality.

Part (3). We consider $a<t \leq b$, then

$$
\frac{\varphi_{1}(|u(t)-u(a)|)}{\kappa((t-a) /(b-a))+\kappa((b-t) /(b-a))} \leq \kappa V_{\phi}(u) .
$$

Then $\varphi_{1}(|u(t)-u(a)|) \leq 2 \kappa V_{\phi}(u)$ and from this inequality we have the required relation.

Part (4). We get Part (4) by the convexity of functions $\varphi_{n}, n \in$ $\mathbb{N}$ and the definition of $\kappa \phi$-variation.

Part (5). It follows from part (4) and Lemma 6.

Part (6). Let $u \in \operatorname{BV}[a, b]$ and consider $\left\{\left[a_{n}, b_{n}\right]\right\}_{n \geq 1} \in$ $F_{\mathbb{N}}[a, b]$. Define

$$
A:=\left\{n \in \mathbb{N}:\left|u\left(b_{n}\right)-u\left(a_{n}\right)\right| \leq 1\right\} .
$$

Then

$$
\begin{aligned}
\sum_{n=1}^{\infty} \varphi_{n}\left(\left|u\left(b_{n}\right)-u\left(a_{n}\right)\right|\right) & \\
\leq & \varphi_{1}(1) \sum_{n \in A}\left|u\left(b_{n}\right)-u\left(a_{n}\right)\right| \\
& +\sum_{n \notin A} \varphi_{n}\left(\left|u\left(b_{n}\right)-u\left(a_{n}\right)\right|\right) .
\end{aligned}
$$

The last sum has at most $[V(u)]$ terms, where $[x]=$ $\max \{n: n \leq x\}$. Because otherwise it has at least $[V(u)]+1$ summands.

Accordingly

$$
V(u) \geq \sum_{n \notin A}\left|u\left(b_{n}\right)-u\left(a_{n}\right)\right| \geq[V(u)]+1 .
$$

Which is a contradiction. Therefore

$$
\begin{aligned}
& \sum_{n=1}^{\infty} \varphi_{n}\left(\left|u\left(b_{n}\right)-u\left(a_{n}\right)\right|\right) \\
& \quad \leq \varphi_{1}(1) V(u)+\sum_{n \notin A} \varphi_{n}\left(\left|u\left(b_{n}\right)-u\left(a_{n}\right)\right|\right) \\
& \quad \leq \varphi_{1}(1) V(u)+\varphi_{1}\left(2\|u\|_{\infty}\right)[V(u)] .
\end{aligned}
$$

This concludes that $\mathrm{BV}[a, b] \subset V_{\phi}[a, b]$.

Let us show that $V_{\phi}[a, b] \subset \kappa V_{\phi}[a, b]$. In Fact let $u \in$ $V_{\phi}[a, b]$ and $\left\{\left[a_{n}, b_{n}\right]\right\}_{n \geq 1} \in F_{\mathbb{N}}[a, b]$, then

$$
\begin{aligned}
& \sum_{n=1}^{\infty} \varphi_{n}\left(\left|u\left(b_{n}\right)-u\left(a_{n}\right)\right|\right) \\
& \quad \leq V_{\phi}(u) \kappa(1) \leq V_{\phi}(u) \kappa\left(\sum_{n=1}^{\infty} \frac{b_{n}-a_{n}}{b-a}\right) .
\end{aligned}
$$

Hence, we get that $\kappa V_{\phi}(u) \leq V_{\phi}(u)$.

Part (7). Let $u \in \kappa \mathrm{BV}[a, b]$, then by part (3) $u$ is bounded in $[a, b]$. Let us fix $\lambda>0$, such that $\|\lambda u\|_{\infty} \leq 1 / 2$. Let 
$\left\{\left[a_{n}, b_{n}\right]\right\}_{n \geq 1} \in F_{\mathbb{N}}[a, b]$, then from the convexity of the functions $\varphi_{n}, n \in \mathbb{N}$, we have

$$
\begin{aligned}
& \frac{\sum_{n=1}^{\infty} \varphi_{n}\left(\left|\lambda u\left(b_{n}\right)-\lambda u\left(a_{n}\right)\right|\right)}{\sum_{n=1}^{\infty} \kappa\left(\left(b_{n}-a_{n}\right) /(b-a)\right)} \\
& \quad \leq \frac{\sum_{n=1}^{\infty} \varphi_{n}(1) \lambda\left|u\left(b_{n}\right)-u\left(a_{n}\right)\right|}{\sum_{n=1}^{\infty} \kappa\left(\left(b_{n}-a_{n}\right) /(b-a)\right)} \\
& \quad \leq \varphi_{1}(1) \lambda \kappa V(u) .
\end{aligned}
$$

Thus Lemma 6 concludes that $u \in \kappa \mathrm{BV}_{\phi}[a, b]$.

Part (8). Suppose that there is $t^{*} \in(a, b]$ such that $\lim _{t \uparrow t^{*}} u(t)$ does not exist.

By part (3) $u$ is bounded then

$$
\alpha=\lim _{t \uparrow t^{*}} \inf u(t)<\lim _{t \uparrow t^{*}} \sup u(t)=\beta, \quad(\alpha, \beta \in \mathbb{R}) .
$$

For each integer $n$ (large enough) we can choose $t_{n}, t_{n}^{\prime}$ such that

$$
\begin{gathered}
t^{*}-\frac{1}{n}<t_{n}<t_{n}^{\prime}<t^{*}, \\
\left|u\left(t_{n}\right)-u\left(t_{n}^{\prime}\right)\right| \geq \beta-\alpha .
\end{gathered}
$$

Using the definition of $\kappa \phi$-variation, we have

$$
\begin{aligned}
& \varphi_{1}\left(\left|u\left(t_{n}\right)-u\left(t_{n}^{\prime}\right)\right|\right) \\
& \times\left(\kappa\left(\frac{t_{n}-a}{b-a}\right)+\kappa\left(\frac{t_{n}^{\prime}-t_{n}}{b-a}\right)+\kappa\left(\frac{b-t_{n}^{\prime}}{b-a}\right)\right)^{-1} \\
& \quad \leq \kappa V_{\phi}(u) .
\end{aligned}
$$

Therefore,

$$
\varphi_{1}(\beta-\alpha) \leq \kappa V_{\phi}(u)\left(2+\kappa\left(\frac{1}{n(b-a)}\right)\right) .
$$

By taking limit when $n \rightarrow \infty$, we obtain $\varphi_{1}(\beta-\alpha)=0$, which is absurd. From each it follows that

$$
\lim _{t \uparrow t^{*}} \inf u(t)=\lim _{t \uparrow t^{*}} \sup u(t) .
$$

By a similar argument it follows that there exist $\lim _{t \downarrow t^{*}} u(t), t^{*} \in[a, b)$.

Assuming that $\lim _{t \rightarrow 0}(k(t) / t)$ is finite, then $\mathrm{V}_{\phi}[a, b]=$ $\kappa V[a, b]$. For the last part of Theorem 9, we can give the definition of left and right regularizations of the function $u \in \kappa V_{\phi}[a, b]$.

Definition 10. Let $u \in \kappa \mathrm{BV}_{\phi}[a, b]$, then

$$
\begin{aligned}
& u^{-}(t):= \begin{cases}\lim _{s \uparrow t} u(s), & t \in(a, b] \\
u(a), & t=a,\end{cases} \\
& u^{+}(t):= \begin{cases}\lim _{s \downarrow t} u(s), & t \in[a, b) \\
u(b), & t=b .\end{cases}
\end{aligned}
$$

The function $u^{-}$is called the left regularization of the function $u$ and the function $u^{+}$the right regularization of the function $u$.

Applying the previous definition and the last part of Theorem 9, we can define

$$
\kappa \mathrm{BV}_{\phi}^{-}[a, b]:=\left\{u^{-}: u \in \kappa \mathrm{BV}_{\phi}[a, b]\right\} .
$$

Similarly, we defined $\kappa \mathrm{BV}_{\phi}^{+}[a, b]$.

Recently Castillo et al. [25] introduced the concept of $\kappa p$ variation in Riesz-Korenblum's sense in the following way.

Definition 11. Let $1<p<\infty, \kappa:[0,1] \rightarrow[0,1]$ be a distortion function and $u:[a, b] \rightarrow \mathbb{R}$ and $\pi: a \leq t_{1}<$ $\cdots<t_{n} \leq b$ a partition of the interval $[a, b]$. We define

$$
\kappa V_{p}^{R}(u)=\kappa V_{p}^{R}(u ;[a, b]):=\sup _{\pi} \kappa \sigma_{p}(u, \pi),
$$

where

$$
\begin{aligned}
\kappa \sigma_{p}(u, \pi):= & \left(\sum_{j=1}^{n-1} \frac{\left|u\left(t_{j+1}\right)-u\left(t_{j}\right)\right|^{p}}{\left|t_{j+1}-t_{j}\right|^{p-1}}\right) \\
& \times\left(\sum_{j=1}^{n-1} \kappa\left(\frac{t_{j+1}-t_{j}}{b-a}\right)\right)^{-1}
\end{aligned}
$$

and the supremum is taken on the set of all partitions of $[a, b]$. If $\kappa V_{p}^{R}(u ;[a, b])<\infty$, we say that $u$ has bounded $\kappa p$-variation in the interval $[a, b]$. The number $\kappa V_{p}^{R}(u ;[a, b])$ denoted the $\kappa p$-variation of $u$ in Riesz-Korenblum's sense in $[a, b]$. The space of functions that have bounded $\kappa p$-variation in the interval $[a, b]$ is denoted by $\kappa \operatorname{RV}_{p}[a, b]$.

Some properties of these functions are exposed in the following theorem.

Theorem 12 (see [25]). Let $1<p<\infty$ and let $\kappa:[0,1] \rightarrow$ $[0,1]$ be a distortion function, then

(1) $\kappa \mathrm{RV}[a, b]$ is a Banach space endowed with the norm

$$
\|u\|_{\kappa p}:=|u(a)|+\left(\kappa V_{p}^{R}(u)\right)^{1 / p} \quad\left(u \in \kappa \mathrm{RV}_{p}[a, b]\right) .
$$

(2) $\operatorname{Lip}[a, b] \subset \operatorname{RV}_{p}[a, b] \subset \kappa \mathrm{RV}_{p}[a, b] \subset \kappa \mathrm{BV}[a, b]$, where $\operatorname{RV}_{p}[a, b]$ denote the space of the functions $u$ that have bounded p-variation in Riesz's sense [20].

(3) $\kappa \mathrm{RV}_{p}[a, b]$ is an algebra.

This concept was generalized by Castillo et al. [26] as stated in the following definition.

Definition 13. Let $\varphi$ be a $\varphi$-function, $\kappa:[0,1] \rightarrow[0,1]$ be a distortion function, and $u:[a, b] \rightarrow \mathbb{R}$ and $\pi: a \leq t_{1}<$ $\cdots<t_{n} \leq b$ a partition of the interval $[a, b]$. We define

$$
\kappa V_{\varphi}^{R}(u)=\kappa V_{\varphi}^{R}(u ;[a, b]):=\sup _{\pi} \kappa \sigma_{\varphi}(u, \pi),
$$


where

$$
\begin{aligned}
& \kappa \sigma_{\varphi}(u, \pi) \\
& \quad:=\frac{\sum_{j=1}^{n-1} \varphi\left(\left|u\left(t_{j+1}\right)-u\left(t_{j}\right)\right| /\left|t_{j+1}-t_{j}\right|\right)\left|t_{j+1}-t_{j}\right|}{\sum_{j=1}^{n-1} \kappa\left(\left(t_{j+1}-t_{j}\right) /(b-a)\right)}
\end{aligned}
$$

and the supremum is taken over all partitions of $[a, b]$. If $\kappa V_{\varphi}^{R}(u ;[a, b])<\infty$, we say that $u$ has bounded $\kappa \varphi$ variation in the interval $[a, b]$ and this number denotes the $\kappa \varphi$-variation of $u$ in Riesz-Korenblum's sense in $[a, b]$. The class of functions that have bounded $\kappa \varphi$-variation in the interval $[a, b]$ is denoted by $\kappa V_{\varphi}[a, b]$. The vectorial space generate by this class is denoted by $\kappa \mathrm{RV}_{\varphi}[a, b]$.

The space of all functions that have bounded $\kappa \varphi$-variation on $[a, b]$ is denoted by $\kappa \operatorname{RV}_{\varphi}[a, b]$. Some properties of these functions are exposed in the following theorem.

Theorem 14 (see [26]). Let $\varphi$ be a convex $\varphi$-function and $\kappa$ : $[0,1] \rightarrow[0,1]$ a distortion function, then

(1) $\operatorname{Lip}[a, b] \subset \kappa V_{\varphi}^{R}[a, b] \subset \kappa \operatorname{BV}[a, b]$.

(2) $\operatorname{RV}_{\varphi}[a, b] \subset \kappa \mathrm{RV}_{\varphi}[a, b] \subset \kappa \mathrm{BV}[a, b]$, where $\mathrm{RV}_{\varphi}[a, b]$ denote the space generated by the class of functions of bounded $\varphi$-variation in Riesz's sense [20].

(3) If $\lim _{t \rightarrow \infty}(\varphi(t) / t)<\infty$, then $\kappa V_{\varphi}^{R}[a, b]=\kappa \mathrm{BV}[a, b]$.

(4) If $u \in \kappa V_{\varphi}^{R}[a, b]$, then $u$ is bounded.

(5) $\kappa V_{\varphi}^{R}[a, b]$ is a convex and symmetric set.

(6) $\kappa \mathrm{RV}_{\varphi}[a, b]=\{u:[a, b] \rightarrow \mathbb{R}: \exists \lambda>0$ such that $\left.\kappa V_{\phi}^{R}(\lambda u)<\infty\right\}$.

(7) $\kappa \mathrm{RV}_{\varphi}[a, b]$ is a Banach space endowed with the norm

$$
\begin{gathered}
\|u\|_{\kappa \varphi}:=|u(a)|+\mu(u) \quad\left(u \in \kappa \operatorname{RV}_{\varphi}[a, b]\right), \\
\text { where } \mu_{\varphi}(u):=\inf _{\lambda>0}\left\{\lambda>0: \kappa V_{\varphi}^{R}(u / \lambda) \leq 1\right\} .
\end{gathered}
$$

\section{Composition Operator between $\operatorname{Lip}[a, b]$ and $\kappa \mathrm{BV}[a, b]$ or $\kappa \mathrm{BV}_{\phi}[a, b]$}

Given a function $h: \mathbb{R} \rightarrow \mathbb{R}$, the composition operator $H$, associated to the function $h$ (case autonomous), maps each function $u:[a, b] \rightarrow \mathbb{R}$ into the composition function $H u$ : $[a, b] \rightarrow \mathbb{R}$ defined by

$$
H u(t):=h(u(t)), \quad(t \in[a, b]) .
$$

More generally, given $h:[a, b] \times \mathbb{R} \rightarrow \mathbb{R}$, we consider operator $H$ defined by

$$
H u(t):=h(t, u(t)), \quad(t \in[a, b]) .
$$

This operator is also called superposition operator or substitution operator or Nemytskii operator. In what follows, will refer to (39) as the autonomous case and to (40) as the nonautonomous case.

A problem related with this operator is to establish necessary and sufficient conditions of function $h$ so that the operators $H$ map the space $\mathbb{X}$ of real functions defined on $[a, b]$ into itself, that is, $H(\mathbb{X}) \subset \mathbb{X}$, or in more general way that operator $H$ maps the space $\mathbb{X}$ into space of functions $\mathbb{Y}(H(\mathbb{X}) \subset \mathbb{Y})$. This problem is sometimes referred to as the composition operator problem (or COP). The solution to this problem for given $\mathbb{X}$ is sometimes very easy and sometimes highly nontrivial. As we mentioned in the introduction of this paper in a variety of spaces the required condition is that function $h$ is locally Lipschitz. Another interesting problem is to determine the smallest space of functions $\mathbb{X}$ and the bigger space $\mathbb{Y}$ such that $H(\mathbb{X}) \subset \mathbb{Y}$.

In order to obtain the main result of this section, we will use a function of the zig-zig type such as the employed by Appell et al. in $[1,15]$. In this section we will show that the locally Lipschitz condition of the function $h$ is a necessary and sufficient condition such that $H(\operatorname{Lip}[a, b]) \subset \kappa \mathrm{BV}[a, b]$ and that in this situation $H$ is bounded.

The following lemma will be useful in the proof of our main theorem (Theorem 17).

Lemma 15. Let $u:[a, b] \rightarrow \mathbb{R}, a \leq s<\eta<t \leq b$, then

$$
\frac{|u(t)-u(s)|}{t-s} \leq \frac{|u(\eta)-u(s)|}{\eta-s}+\frac{|u(t)-u(\eta)|}{t-\eta} .
$$

Proof. Let $a \leq s<\eta<t \leq b$. Then

$$
\begin{aligned}
& \frac{|u(t)-u(s)|}{t-s} \\
& \quad \leq \frac{|u(\eta)-u(s)|}{t-s}+\frac{|u(t)-u(\eta)|}{t-s} \\
& =\frac{|u(\eta)-u(s)|}{\eta-s}\left(\frac{\eta-s}{t-s}\right)+\frac{|u(t)-u(\eta)|}{t-\eta}\left(\frac{t-\eta}{t-s}\right) \\
& \quad \leq \frac{|u(\eta)-u(s)|}{\eta-s}+\frac{|u(t)-u(\eta)|}{t-\eta} .
\end{aligned}
$$

Lemma 16. Let $\kappa:[0,1] \rightarrow[0,1]$ be a distortion function, $\phi=\left\{\varphi_{n}\right\}_{n \geq 1}$ a $\phi$-sequence, $u \in \kappa \mathrm{BV}_{\phi}[a, b]$, and $\lambda>0$. Then $\mu_{\phi}(u)<\lambda$ if and only if $\kappa V_{\phi}(u / \lambda)<1$.

Proof. Let $u \in \kappa \mathrm{BV}_{\phi}[a, b]$. Suppose that $\mu_{\phi}(u)<\lambda$; then, by definition of $\mu_{\phi}(u)$ there exists $k$ such that $\lambda>k>\mu_{\phi}(u)$ and $\kappa V_{\phi}(u / k) \leq 1$. Hence, by the convexity of the functions $\varphi_{n}$, we have

$$
\kappa V_{\phi}\left(\frac{u}{\lambda}\right)=\kappa V_{\phi}\left(\frac{u}{k} \frac{k}{\lambda}\right) \leq \frac{k}{\lambda} \kappa V_{\phi}\left(\frac{u}{k}\right) \leq \frac{k}{\lambda} \leq 1 .
$$

Conversely, assume $\kappa V_{\phi}(u / \lambda)<1$, then $\lambda \in\{\lambda>0$ : $\left.\kappa V_{\phi}(u / \lambda) \leq 1\right\}$; hence $\mu_{\phi}(u)<\lambda$.

Theorem 17. Let $\kappa:[0,1] \rightarrow[0,1]$ be a distortion function, $\phi=\left\{\varphi_{n}\right\}_{n \geq 1}$ a $\phi$-sequence, $h: \mathbb{R} \rightarrow \mathbb{R}$, and $H$ the composition 
operator associated to $h$. $H$ maps the space Lip $[0,1]$ into the space $\kappa \mathrm{BV}_{\phi}[0,1]$ or $\kappa \mathrm{BV}[0,1]$ if and only if $h$ is locally Lipschitz. Furthermore operator $H$ is bounded.

Proof. Let $u \in \operatorname{Lip}[0,1], r=\|u\|_{\infty}$ and suppose that $h$ is locally Lipschitz, then there exist $k=k(r)$, such that

$$
|h(t)-h(s)| \leq k(r)|s-t|, \quad(s, t \in \mathbb{R},|s| \leq r,|t| \leq r) .
$$

Let $\left\{\left[a_{n}, b_{n}\right]\right\}_{n \geq 1} \in F_{\mathbb{N}}[a, b]$ and $\lambda>0$, such that $\lambda<$ $1 /\left(2 k(r)\|u\|_{\text {Lip }}\|u\|_{\infty}+1\right)$, then

$$
\begin{aligned}
& \frac{\sum_{n=1}^{\infty} \varphi_{n}\left(\lambda\left|h\left(u\left(b_{n}\right)\right)-h\left(u\left(a_{n}\right)\right)\right|\right)}{\sum_{n=1}^{\infty} \kappa\left(\left(b_{n}-a_{n}\right) /(b-a)\right)} \\
& \quad \leq \frac{\sum_{n=1}^{\infty} \varphi_{n}\left(\lambda k(r)\left|u\left(b_{n}\right)-u\left(a_{n}\right)\right|\right)}{\kappa(1)} \\
& \quad \leq \lambda k(r) \varphi_{1}(1)\|u\|_{\text {Lip }} \sum_{n=1}^{\infty}\left(b_{n}-a_{n}\right)<\infty .
\end{aligned}
$$

Then by Lemma 15 and Theorem 9, we have $H(u) \in$ $\kappa \mathrm{BV}_{\phi}[a, b]$.

The proof of the only if direction will be by contradiction, that is, we assume $H(\operatorname{Lip}[0,1]) \subset \kappa \mathrm{BV}_{\phi}[0,1]$ and $h$ is not locally Lipschitz. Since the identity function $I_{d}:[0,1] \rightarrow$ $[0,1]$ belongs to $\operatorname{Lip}[0,1]$, then $h \circ I_{d} \in \kappa \mathrm{BV}_{\phi}[0,1]$ and therefore $h$ is bounded in the interval $[0,1]$. Without loss of generality we may assume that

$$
\left\|\left.h\right|_{[0,1]}\right\|_{\infty} \leq \frac{1}{4} .
$$

Since $h$ is not locally Lipschitz in $\mathbb{R}$, there is a closed interval $I$ such that $h$ does not satisfy any Lipschitz condition. In order to simplify the proof we can assume that $I=[0,1]$. In this way for any increasing sequence of positive real numbers $\left\{k_{n}\right\}_{n \geq 1}$ that converge to infinite that we will define later, we can choose sequences $\left\{a_{n}\right\}_{n \geq 1},\left\{b_{n}\right\}_{n \geq 1}$, such that

$$
\left|h\left(b_{n}\right)-h\left(a_{n}\right)\right|>k_{n}\left|b_{n}-a_{n}\right|, \quad(n \in \mathbb{N}) .
$$

In addition we can choose $a_{n}, b_{n}$ such that

$$
a_{n}<b_{n}, \quad(n \in \mathbb{N}) \text {. }
$$

Considering subsequences if necessary, we can assume that the sequence $\left\{a_{n}\right\}_{n \geq 1}$ is monotone. We can assume without loss of generality that sequence $\left\{a_{n}\right\}_{n>1}$ is increasing.

Since $[0,1]$ is compact, from inequality $(47)$ we have that there exist subsequences of $\left\{a_{n}\right\}_{n \geq 1}$ and $\left\{b_{n}\right\}_{n \geq 1}$ that we will denote in the same way, and that converge to $a_{\infty} \in[0,1]$.

Since the sequence $\left\{a_{n}\right\}_{n \geq 1}$ is a Cauchy sequence, we can assume (taking subsequence if necessary) that

$$
\left|a_{m}-a_{n}\right|<\frac{1}{k_{n}}, \quad(m>n) .
$$

Again considering subsequences if needed using the properties of the function $\kappa$ we can assume that

$$
\max \left\{\kappa\left(b_{n}-a_{n}\right), \kappa\left(a_{m}-a_{n}\right)\right\}<\frac{1}{k_{n}}, \quad(n \in \mathbb{N}, m \geq n) .
$$

Consider the new sequence $\left\{m_{n}\right\}_{n \geq 1}$ defined by

$$
m_{n}:=\frac{1}{k_{n}\left(b_{n}-a_{n}\right)}, \quad(n \in \mathbb{N}) .
$$

From inequalities (46) and (47) it follows that $m_{n}>2$; therefore

$$
\frac{m_{n}}{2}<\left[m_{n}\right] \leq m_{n}, \quad(n \in \mathbb{N}) .
$$

Consider the sequence defined recursively $\left\{t_{n}\right\}_{n \geq 1}$ by

$$
\begin{array}{r}
t_{1}:=0, \quad t_{n+1}:=t_{n}+a_{n+1}-a_{n}+2\left[m_{n}\right]\left(b_{n}-a_{n}\right), \\
(n \in \mathbb{N}) .
\end{array}
$$

This sequence is strictly increasing and from the relations (49) and (50), we get

$$
\begin{aligned}
t_{n} \longrightarrow t_{\infty} & :=\sum\left(t_{n+1}-t_{n}\right) \\
& =\sum_{n=1}^{\infty}\left(a_{n+1}-a_{n}\right)+2 \sum_{n=1}^{\infty}\left[m_{n}\right]\left(b_{n}-a_{n}\right) \\
& \leq 3 \sum_{n=1}^{\infty} \frac{1}{k_{n}} .
\end{aligned}
$$

Then to ensure that $t_{\infty} \in[0,1]$, it is sufficient to suppose that $\sum_{n=1}^{\infty}\left(1 / k_{n}\right) \leq 1 / 3$.

We define the continuous zig-zag function $u:[0,1] \rightarrow$ $\mathbb{R}$, as shown in the following:

$u(t)$

$:= \begin{cases}a_{n}, & t=t_{n}+2 i\left(b_{n}-a_{n}\right), i=0, \ldots,\left[m_{n}\right] \\ b_{n}, & t=t_{n}+(2 i+1)\left(b_{n}-a_{n}\right), i=0, \ldots,\left[m_{n}\right]-1 \\ a_{\infty}, & t_{\infty} \leq t \leq 1 \\ \text { affine, } & \text { other case. }\end{cases}$

Put

$$
t_{n, i}:=t_{n}+i\left(b_{n}-a_{n}\right), \quad n \in \mathbb{N}, i=0, \ldots, 2\left[m_{n}\right] .
$$

We can write each interval $I_{n}=\left[t_{n}, t_{n+1}\right], n \in \mathbb{N}$, as the union of the family of nonoverlapping intervals

$$
\begin{aligned}
& I_{n, i}:=\left[t_{n . i}, t_{n, i+1}\right], \quad i=0, \ldots,\left[m_{n}\right]-1, \\
& I_{n, 2\left[m_{n}\right]}:=\left[t_{n, 2\left[m_{n}\right]}, t_{n+1}\right] .
\end{aligned}
$$

And function $u$ is defined on $I_{n, i}, i=0, \ldots, 2\left[m_{n}\right]$ as follows:

$$
\begin{gathered}
u(t)=t-\left(t_{n}+2 i\left(b_{n}-a_{n}\right)\right)+a_{n}, \quad\left(t \in I_{n, 2 i}\right), \\
u(t)=t-t_{n}+(2 i+1)\left(b_{n}-a_{n}\right)+b_{n}, \quad\left(t \in I_{n, 2 i+1}\right), \\
u(t)=t-t_{n+1}+a_{n}, \quad\left(t \in I_{n, 2[m]}\right) .
\end{gathered}
$$

In all these situations, the slopes of the segments of lines are equal to 1 . 
Hence, we have for $n \in \mathbb{N}$, the absolute value of the slope of the line segments in these ranges are bounded by 1 , as shown below

$$
\begin{gathered}
2^{-n} \frac{\left|b_{n}-a_{n}\right|}{\kappa^{-1}\left(b_{n}-a_{n}\right)} \leq 2^{-n} k_{n}\left(b_{n}-a_{n}\right) \leq 1, \\
2^{-(n+1)} \frac{a_{n+1}-a_{n}}{t_{n}+a_{n+1}-a_{n}} \leq 1 .
\end{gathered}
$$

We will show that $u \in \operatorname{Lip}[0,1]$.

Let $0 \leq s<t \leq 1$, and then there are the following possibilities for the location of $s$ and $t$ on $[0,1]$.

Case 1. If $s, t \in I_{n},(n \in \mathbb{N})$ and are in the same interval $I_{n, i}, \quad i=0, \ldots, 2\left[m_{n}\right]$.

From relations (58), (59), and (60) it follows that $\mid u(t)-$ $u(s)|/| t-s \mid=1$.

Case 2. If $s, t \in I_{n}, \quad(n \in \mathbb{N})$ and are in two different intervals $I_{n, i}, \quad i=0, \ldots, 2\left[m_{n}\right]$.

There are several possibilities.

(a) $s \in I_{n . i}, t \in I_{n, j}, i<j<2\left[m_{n}\right]$.

$\left(\mathrm{a}_{1}\right) j=i+1$. By Lemma 15 and relations (58) and (59) we have

$$
\begin{aligned}
& \frac{\mid u(t)}{|t-s|} \\
& \quad \leq \frac{\left|u\left(t_{n, i+1}\right)-u(s)\right|}{t_{n, i+1}-s}+\frac{\left|u(t)-u\left(t_{n, i+1}\right)\right|}{t-t_{n, i+1}} \leq 2 .
\end{aligned}
$$

$\left(\mathrm{a}_{2}\right) j>i+1$. Then

$$
\frac{|u(t)-u(s)|}{|t-s|} \leq \frac{b_{n}-a_{n}}{t_{n, i+2}-t_{n, i+1}}=1 .
$$

(b) $s \in I_{n . i}, \quad t \in I_{n, j}, \quad i<j=2\left[m_{n}\right]$.

If $j=i+1$, proceed as $\left(a_{1}\right)$.

If $j>i+1$, again using the Lemma 15 and relations (58), (59), and (60) we obtain

$$
\begin{aligned}
& \frac{\mid u(t)}{\mid t}-\boldsymbol{u}(s) \mid \\
& \quad \leq \frac{\left|u\left(t_{n, 2\left[m_{n}\right]}\right)-u(s)\right|}{t_{n, 2\left[m_{n}\right]}-s}+\frac{\left|u(t)-u\left(t_{n, 2\left[m_{n}\right]}\right)\right|}{t_{n, 2\left[m_{n}\right]}-t} \\
& \quad \leq \frac{b_{n}-a_{n}}{t_{n, 2\left[m_{n}\right]}-t_{n, 2\left[m_{n}\right]-1}}+1 \leq 2 .
\end{aligned}
$$

Case 3. If $s \in I_{n}, t \in I_{m}, n, m \in \mathbb{N}, n<m$.

From Lemma 15 and Case 2, we conclude that

$$
\begin{aligned}
& \frac{|u(t)-u(s)|}{t}-s \\
& \quad \leq \frac{\left|u\left(t_{n+1}\right)-u(s)\right|}{t_{n+1}-s}+\frac{\left|u(t)-u\left(t_{m}\right)\right|}{t-t_{m}} \leq 4 .
\end{aligned}
$$

Case 4. If $s \in I_{n}, n \in \mathbb{N}, t=t_{\infty}$.

Then from Lemma 15

$$
\begin{aligned}
& \frac{\left|u\left(t_{\infty}\right)-u(s)\right|}{t_{\infty}-s} \\
& \quad \leq \frac{\left|u\left(t_{n, i+1}\right)-u(s)\right|}{t_{n, i+1}-s}+\frac{\left|a_{\infty}-u\left(t_{n, i+1}\right)\right|}{b_{n}-a_{n}} \\
& \quad \leq 1+\frac{a_{\infty}-a_{n}}{b_{n}-a_{n}} \leq 2 .
\end{aligned}
$$

Case 5. If $s<t_{\infty}<t \leq 1$.

From Lemma 15 and Case 4,

$$
\frac{|u(t)-u(s)|}{t-s} \leq \frac{\left|u\left(t_{\infty}\right)-u(s)\right|}{t_{\infty}-s} \leq 2 .
$$

Case 6. If $t_{\infty} \leq s<t \leq 1$.

In this circumstance $u(s)=u(t)=a_{\infty}$ and the situation is trivial. Therefore we have that

$$
|u(t)-u(s)| \leq|t-s|, \quad(s, t \in[0,1]) .
$$

So $u$ is Lipschitz in $[0,1]$. Moreover, for each partition of the interval $[0,1]$ of the form

$$
\begin{aligned}
\pi: 0 & =t_{1}<t_{1}+\left(b_{1}-a_{1}\right)<\cdots<t_{1}+2\left[m_{1}\right]\left(b_{1}-a_{2}\right) \\
& <t_{2}<t_{2}+\left(b_{2}-a_{2}\right)<\cdots<t_{k} \\
& <\cdots<t_{k}+2\left[m_{k}\right]\left(b_{k}-a_{k}\right)<1
\end{aligned}
$$

and $c>0$, using the inequality (47), convexity of the function $\varphi_{n}, n \geq 1$, and definition of $m_{n}, n \in \mathbb{N}$, we have

$$
\begin{aligned}
\kappa V_{\phi}(c(h \circ u) ;[0,1]) \\
\quad \geq \frac{\sum_{n=1}^{k} 2\left[m_{n}\right] \varphi_{n}(c)\left|h\left(b_{n}\right)-h\left(a_{n}\right)\right|}{\sum_{n=1}^{k}\left[2\left[m_{n}\right] \kappa\left(b_{n}-a_{n}\right)+\kappa\left(a_{n+1}-a_{n}\right)\right]} \\
\geq \frac{\sum_{n=1}^{k} 2\left[m_{n}\right] \varphi_{n}(c) k_{n}\left(b_{n}-a_{n}\right)}{\sum_{n=1}^{k}\left[2\left[m_{n}\right] \kappa\left(b_{n}-a_{n}\right)+\kappa\left(a_{n+1}-a_{n}\right)\right]} \\
\geq \frac{\sum_{n=1}^{k} 2\left[m_{n}\right] k_{n}\left(b_{n}-a_{n}\right) \varphi_{n}(c)}{\sum_{n=1}^{k}\left(1 / k_{n}\right)} \geq \sum_{n=1}^{k} \varphi_{n}(c) .
\end{aligned}
$$

As the series $\sum_{n=1}^{\infty} \varphi_{n}(c)$ diverge, $c h \circ u \notin \kappa \mathrm{BV}_{\phi}[0,1]$, which is a contradiction.

Let us see that $h \circ u \notin \kappa \mathrm{BV}[a, b]$. In fact, as in the case of $\kappa$-variation we have

$$
V_{\kappa}(h \circ u ;[0,1]) \geq \sum_{n=1}^{k} 2\left[m_{k}\right] k_{n}\left(b_{n}-a_{n}\right) \geq k .
$$

Therefore, $h \circ u \notin \kappa \mathrm{BV}[0,1]$. 
To prove that the operator $H$ is bounded, let $r>0$ and $u \in \operatorname{Lip}[0,1]$, such that $\|u\|_{\text {Lip }} \leq r$, then from the definition of $\|\cdot\|_{\text {Lip }[0,1]}$, we have

$$
\|u\|_{\infty} \leq|u(0)|+L_{0}^{1}(u) \leq r .
$$

Without loss of generality assume that $r=1$. As $h$ is locally Lipschitz, there exists $k(1)>0$, such that

$$
|h(x)-h(y)| \leq k(1)|x-y|, \quad|x| \leq 1,|y| \leq 1 .
$$

As the identity function $I_{d}:[0,1] \rightarrow[0,1]$ belongs to $\operatorname{Lip}[0,1]$, then $h \circ I_{d} \in \kappa \mathrm{BV}_{\phi}[0,1]$. By Theorem 9 we have that $\left\|h \circ I_{d}\right\|_{\infty}<\infty$.

Let $\left\{\left[a_{n}, b_{n}\right]\right\}_{n \geq 1} \in F_{\mathbb{N}}[0,1]$ and choose $\lambda$ such that $k(1) \kappa V_{\phi}\left(I_{d}\right)<\lambda$, then

$$
\begin{aligned}
\frac{\sum_{n=1}^{\infty} \varphi_{n}\left(\left|h\left(u\left(b_{n}\right)\right)-h\left(u\left(a_{n}\right)\right)\right| / \lambda\right)}{\sum_{n=1}^{\infty} \kappa\left(b_{n}-a_{n}\right)} & \\
\leq & \sum_{n=1}^{\infty} \varphi_{n}\left(\frac{k(1)\left|u\left(b_{n}\right)-u\left(a_{n}\right)\right|}{\lambda}\right) \\
\leq & \sum_{n=1}^{\infty} \varphi_{n}\left(\frac{k(1)\left(b_{n}-a_{n}\right)}{\lambda}\right) \\
\leq & \sum_{n=1}^{\infty} \frac{k(1)}{\lambda} \varphi_{n}\left(b_{n}-a_{n}\right) \\
\leq & \frac{k(1)}{\lambda} \kappa V_{\phi}\left(I_{d}\right)<1 .
\end{aligned}
$$
that

From Lemma 6 we have $\mu(h \circ u)<\lambda(r)$. Thus we conclude

$$
\begin{aligned}
\|H(u)\|_{\kappa \phi} & =|h(u(a))|+\mu(h \circ u) \\
& \leq\|h \circ u\|_{\infty}+\lambda(r) .
\end{aligned}
$$

And so operator $H$ is bounded. In the case that $H(\operatorname{Lip}[0,1]) \subset \kappa \mathrm{BV}[0,1]$, proceed similarly.

In the following result we give a Lemma of invariance.

Lemma 18. Let $\kappa$ be a distortion function, $\phi=\left\{\varphi_{n}\right\}_{n \geq 1} a \phi$ sequence, and $v:[0,1] \rightarrow[a, b]$ affine function that maps $[0,1]$ on $[a, b](v(t):=(b-a) t+a, t \in[0,1])$.

(1) $u \in \kappa \mathrm{BV}_{\phi}[a, b]$ if and only if $u \circ \nu \in \kappa \mathrm{BV}_{\phi}[0,1]$.

(2) $u \in \kappa \mathrm{BV}[a, b]$ if and only if $u \circ v \in \kappa \mathrm{BV}_{\phi}[0,1]$.

Proof. (1) Let $u \in \kappa \mathrm{BV}_{\phi}[a, b]$ and $\left\{\left[a_{n}, b_{n}\right]\right\}_{n \geq 1} \in F_{\mathbb{N}}[0,1]$, then $\left\{\left[\nu\left(a_{n}\right), v\left(b_{n}\right)\right]\right\}_{n \geq 1} \in F_{\mathbb{N}}[a, b]$ and

$$
\begin{aligned}
& \frac{\sum_{n=1}^{\infty}\left|u\left(\nu\left(b_{n}\right)\right)-u\left(\nu\left(a_{n}\right)\right)\right|}{\sum_{n=1}^{\infty} \kappa\left(b_{n}-a_{n}\right)} \\
& =\frac{\sum_{n=1}^{\infty}\left|u\left(\nu\left(b_{n}\right)\right)-u\left(\nu\left(a_{n}\right)\right)\right|}{\sum_{n=1}^{n-1} \kappa\left(\left(\nu\left(b_{n}\right)-\nu\left(a_{n}\right)\right) /(b-a)\right)} \\
& \leq \kappa V_{\phi}(u ;[a, b]) .
\end{aligned}
$$

Hence $u \circ v \in \kappa \mathrm{BV}_{\phi}[0,1]$.

Reciprocally, let us suppose that $u \circ v \in \kappa \mathrm{BV}_{\phi}[0,1]$ and let $\left\{\left[a_{n}, b_{n}\right]\right\}_{n \geq 1} \in F_{\mathbb{N}}[a, b]$ be a partition of the interval $[a, b]$. Define

$$
I_{n}:=\left[\nu^{-1}\left(b_{n}\right), \nu^{-1}\left(a_{n}\right)\right] \quad(n \in \mathbb{N}) .
$$

We have $\left\{I_{n}\right\}_{n \geq 1} \in F_{\mathbb{N}}[0,1]$ and

$$
\begin{aligned}
& \frac{\sum_{j=1}^{n-1}\left|u\left(b_{n}\right)-u\left(a_{n}\right)\right|}{\sum_{n=1}^{\infty} \kappa\left(\left(b_{n}-a_{n}\right) /(b-a)\right)} \\
& =\left(\sum_{n=1}^{\infty} \mid u\left(\nu\left(\left(b_{n}-a\right) /(b-a)\right)\right)\right. \\
& \left.-u\left(\nu\left(\left(a_{n}-a\right) /(b-a)\right)\right) \mid\right) \\
& \times\left(\sum _ { n = 1 } ^ { \infty } \kappa \left(\left(b_{n}-a\right) /(b-a)\right.\right. \\
& \left.\left.-\left(a_{n}-a\right) /(b-a)\right)\right)^{-1} \\
& \leq \kappa V_{\phi}(u \circ v ;[0,1]),
\end{aligned}
$$

so $u \in \kappa \mathrm{BV}_{\phi}[a, b]$.

(2) The proof is similar from part (1).

As consequence of Lemma 18 we have the following results

Lemma 19. Let $\kappa$ be a distortion function, $\phi=\left\{\varphi_{n}\right\}_{n \geq 1} a$ $\phi$-sequence, $h: \mathbb{R} \rightarrow \mathbb{R}$, and $H$ the composition operator associated to the function $h$.

(1) $H(\operatorname{Lip}[a, b]) \subset \kappa \mathrm{BV}_{\phi}[a, b]$ if and only if $H(\operatorname{Lip}[0,1]) \subset$ $\kappa \mathrm{BV}_{\phi}[0,1]$.

(2) $H(\operatorname{Lip}[a, b]) \subset \kappa \mathrm{BV}[a, b]$ if and only if $H(\operatorname{Lip}[0,1]) \subset$ $\kappa \mathrm{BV}[0,1]$.

Corollary 20. Let $\kappa$ be a distortion function, $\phi=\left\{\varphi_{n}\right\}_{n>1} a \phi$ sequence, and $h: \mathbb{R} \rightarrow \mathbb{R}$. Then the composition operator $H$, associated with function $h$, maps the space $\operatorname{Lip}[a, b]$ on $\kappa \mathrm{BV}_{\phi}[a, b]$ or on $\kappa \mathrm{BV}[a, b]$ if and only if $h$ is locally Lipschitz.

Corollary 21. Let $\kappa$ be a distortion function, $\phi=\left\{\varphi_{n}\right\}_{n \geq 1} a$ $\phi$-sequence, $h: \mathbb{R} \rightarrow \mathbb{R}$, and $\mathbb{X}, \mathbb{Y}$ normed spaces such that $\operatorname{Lip}[a, b] \subset \mathbb{X} \subset \mathbb{Y}$, where $\mathbb{Y} \subset \kappa \mathrm{BV}_{\phi}[a, b]$ or $\mathbb{Y} \subset \kappa \mathrm{BV}[a, b]$. Then the composition operator $H$ associated with the function $h$ maps the space $\mathbb{X}$ in the space $\mathbb{Y}$ if and only if $h$ is locally Lipschitz.

Some particular cases of Corollary 21 are the following

(1) $\mathbb{X}=\mathbb{Y}$, where $\mathbb{X}$ is one of the following spaces $\operatorname{Lip}[a, b][3], H_{\alpha}[a, b](0<\alpha<1)[4], \operatorname{BV}_{\varphi}[a, b][6]$, $\operatorname{BV}[a, b][5], \mathrm{HBV}[7], \operatorname{AC}[a, b][8], \operatorname{RV}_{\varphi}[a, b][9]$, $\phi \mathrm{BV}[23], \Lambda \mathrm{BV}[11]$, and $\kappa \mathrm{BV}[a, b][12]$. 
(2) $\mathbb{X}=\mathrm{RV}_{\varphi}[a, b], \mathbb{Y}=\mathrm{BV}[a, b]$. See $[10]$.

(3) $\mathbb{X}=\operatorname{Lip}[a, b], \mathbb{Y}=\operatorname{BV}[a, b]$. See $[1]$.

From Corollary 21 we get the following new cases

(1) $\mathbb{X}$ is one of the following spaces: $\operatorname{Lip}[a, b], H_{\alpha}(0<$ $\alpha<1), \operatorname{RV}_{\varphi}[a, b], \mathrm{AC}[a, b], \mathrm{BV}[a, b], \mathrm{BV}_{\phi}[a, b]$, and $\kappa \operatorname{RV}_{\varphi}[a, b] ; \mathbb{Y}$ is one of the following spaces: $\kappa \mathrm{BV}[a, b], \kappa \mathrm{BV}_{\phi}[a, b]$.

(2) $\mathbb{X}=\kappa \mathrm{RV}_{p}[a, b], \mathbb{Y}=\kappa \mathrm{RV}_{q}[a, b], 1<p, q<\infty$.

(3) $\mathbb{X}=\kappa \mathrm{BV}[a, b], \mathbb{Y}=\kappa \mathrm{BV}_{\phi}[a, b]$,

More generally

(1) $\mathbb{X}=\kappa_{1} \mathrm{BV}[a, b], \mathbb{Y}=\kappa_{2} \operatorname{RV}_{\phi}[a, b]$;

(2) $\mathbb{X}=\kappa_{1} \mathrm{RV}_{\varphi}[a, b], \mathbb{Y}=\kappa_{2} \operatorname{RV}_{\psi}[a, b]$;

(3) $\mathbb{X}=\kappa_{1} \operatorname{RV}_{\phi}[a, b], \mathbb{Y}=\kappa_{2} \operatorname{RV}_{\phi}[a, b]$;

where $\kappa_{1}, \kappa_{2}$ are distortion functions.

\section{Uniformly Continuous Composition Operator in the Space $\kappa \mathrm{BV}_{\phi}[a, b]$}

In many problems solving equation where the composition operator appears to guarantee the existence of solution it is necessary to apply a Fixed Point Theorem. To ensure the application this type of results is necessary to request the condition of global Lipschitz operator $H$. In several works Matkowski and Mís have shown that this condition implies that the function $h$ has the form (1) or (2) (see, e.g., $[16,27]$ ). This means that we may apply the Banach contraction mapping principle only if the underlying problems are actually linear and therefore are not interesting.

More recently, Matkowski and other researchers have replaced the condition of global Lipschitz by uniform continuity conditions or uniform boundedness composition operator (see e.g., [14]).

In this section we present results in this direction for the space $\kappa \mathrm{BV}_{\phi}[a, b]$.

Theorem 22. Let $\kappa:[0,1] \rightarrow[0,1]$ be a distortion function, $\phi=\left\{\varphi_{n}\right\}_{n \geq 1} a \phi$-sequence, and $h:[a, b] \times \mathbb{R} \rightarrow \mathbb{R}$ such that the function $h(t, \cdot): \mathbb{R} \rightarrow \mathbb{R}$ is continuous with respect to the second variable, for each $t \in(a, b]$. If the composition operator $H$, associated with $h$, maps $\kappa \mathrm{BV}_{\phi}[a, b]$ into itself and satisfies the inequality

$$
\begin{array}{r}
\|H(u)-H(v)\|_{\kappa \phi} \leq \gamma\left(\|u-v\|_{\kappa \phi}\right), \\
\left(u, v \in \kappa \mathrm{BV}_{\phi}[a, b]\right),
\end{array}
$$

then there exists $\alpha, \beta \in \kappa \mathrm{BV}_{\phi}^{-}[a, b]$, such that

$$
h^{-}(t, x)=\alpha(t) x+\beta(t), \quad(t \in[a, b], x \in \mathbb{R}) .
$$

Proof. As for each $x \in \mathbb{R}$ fixed the function $u(t)=x, t \in[a, b]$ is in $\kappa \mathrm{BV}_{\phi}[a, b]$, then $H(u)=h(\cdot, x) \in \kappa \mathrm{BV}_{\phi}[a, b]$. There is left regularization of $h^{-}(\cdot, x)$.
From inequality (79) and Lemma 16, we get

$$
\kappa V_{\phi}\left(\frac{H(u)-H(v)}{\gamma\left(\|u-v\|_{\kappa \phi}\right)}\right)<1, \quad\left(u, v \in \kappa \mathrm{BV}_{\phi}[a, b]\right) .
$$

Let $a<r<a_{1}<b_{1}<a_{2}<\cdots<a_{m}<b_{m}=$ $s<b, x_{1}, x_{2} \in \mathbb{R}, x_{1} \neq x_{2}$ and put the zig-zag continuous functions $u_{k}:[a, b] \rightarrow \mathbb{R}, k=1,2$, as

$u_{k}(t)$

$$
= \begin{cases}\frac{x_{k}+x_{2}}{2}, & a \leq t \leq a_{1} \text { or } t=a_{i}, i=1, \ldots, m \\ \frac{x_{k}+x_{1}}{2}, & b_{m} \leq t \leq b \text { or } t=b_{i}, i=1, \ldots, m, k=1,2 \\ \text { affine, } & \text { other case. }\end{cases}
$$

The functions $u_{k}, k=1,2$ are Lipschitz and therefore belongs to $\kappa \mathrm{BV}_{\phi}[a, b]$. Furthermore

$$
\left(u_{1}-u_{2}\right)(t)=\frac{x_{1}-x_{2}}{2}, \quad(t \in[a, b]) .
$$

From inequality (81) and the definition of $k \phi$-variation, we have

$$
\begin{gathered}
\left(\sum _ { n = 1 } ^ { m } \varphi _ { n } \left(\left(\mid h\left(b_{n}, u_{1}\left(b_{n}\right)\right)-h\left(b_{n}, u_{2}\left(b_{n}\right)\right)\right.\right.\right. \\
\left.-h\left(a_{n}, u_{1}\left(a_{n}\right)\right)+h\left(a_{n}, u_{2}\left(a_{n}\right)\right) \mid\right) \\
\left.\left.\times\left(\gamma\left(\left\|u_{1}-u_{2}\right\|_{\kappa \phi}\right)\right)^{-1}\right)\right) \\
\times\left(\kappa\left(\frac{a_{1}-a}{b-a}\right)+\sum_{n=1}^{m} \kappa\left(\frac{b_{n}-a_{n}}{b-a}\right)\right. \\
\left.+\sum_{n=1}^{m-1} \kappa\left(\frac{a_{n+1}-b_{n}}{b-a}\right)+\kappa\left(\frac{b-b_{m}}{b-a}\right)\right)^{-1} \leq 1 .
\end{gathered}
$$

By the construction of the $u_{k}, k=1,2$, we get

$$
\begin{gathered}
\left(\sum _ { n = 1 } ^ { m } \varphi _ { n } \left(\left(2 h\left(b_{n}, x_{1}\right)-h\left(b_{n}, \frac{x_{1}+x_{2}}{2}\right)\right.\right.\right. \\
\left.-h\left(a_{n}, \frac{x_{1}+x_{2}}{2}\right)+h\left(a_{n}, x_{2}\right)\right) \\
\left.\left.\times\left(\gamma\left(2^{-1}\left|x_{1}-x_{2}\right|\right)\right)^{-1}\right)\right) \\
\times\left(\kappa\left(\frac{a_{1}-a}{b-a}\right)+\sum_{n=1}^{m} \kappa\left(\frac{b_{n}-a_{n}}{b-a}\right)\right. \\
\left.+\sum_{n=1}^{m-1} \kappa\left(\frac{a_{n+1}-b_{n}}{b-a}\right)+\kappa\left(\frac{b-b_{m}}{b-a}\right)\right)^{-1} \leq 1 .
\end{gathered}
$$


Let $r$ tend to $s$ in the above inequality; we obtain

$$
\begin{aligned}
& \sum_{n=1}^{m} \varphi_{n}\left(\frac{\left|h^{-}\left(s, x_{1}\right)-2 h^{-}\left(s,\left(x_{1}+x_{2}\right) / 2\right)+h^{-}\left(s, x_{2}\right)\right|}{\gamma\left(2^{-1}\left|x_{1}-x_{2}\right|\right)}\right) \\
& \quad \leq \kappa\left(\frac{s-a}{b-a}\right)+\kappa\left(\frac{b-s}{b-a}\right), \quad(s \in(a, b]) .
\end{aligned}
$$

Passing the limit as $m \rightarrow \infty$,

$$
\begin{aligned}
& \sum_{n=1}^{\infty} \varphi_{n}\left(\frac{\left|h^{-}\left(s, x_{1}\right)-2 h^{-}\left(s,\left(x_{1}+x_{2}\right) / 2\right)+h^{-}\left(s, x_{2}\right)\right|}{\gamma\left(2^{-1}\left|x_{1}-x_{2}\right|\right)}\right) \\
& \quad \leq \kappa\left(\frac{s-a}{b-a}\right)+\kappa\left(\frac{b-s}{b-a}\right), \quad(s \in(a, b]) .
\end{aligned}
$$

As the series $\sum_{n=1}^{\infty} \varphi_{n}(x)$ is divergent for each $x>0$, necessarily

$$
\begin{array}{r}
\left|h^{-}\left(s, x_{1}\right)-2 h^{-}\left(s, \frac{x_{1}+x_{2}}{2}\right)+h^{-}\left(s, x_{2}\right)\right|=0, \\
(s \in(a, b]) .
\end{array}
$$

So we conclude that $h^{-}(s, \cdot)$ satisfies the Jensen equation in $\mathbb{R}$ (see [28], page 315). The continuity of $h$ with respect of the second variable implies that for every $t \in[a, b]$ there exist $\alpha, \beta:[a, b] \rightarrow \mathbb{R}$, such that

$$
h^{-}(t, x)=\alpha(t) x+\beta(t), \quad(t \in[a, b], x \in \mathbb{R}) .
$$

Since $\beta(t)=h^{-}(t, 0), t \in[a, b], \alpha(t)=h^{-}(t, 1)-\beta(t)$ and $h^{-}(\cdot, x) \in \kappa \mathrm{BV}_{\phi}[a, b]$, for each $x \in \mathbb{R}$, we obtain that $\alpha, \beta \in \kappa \mathrm{BV}_{\phi}[a, b]$.

Corollary 23. Let $\kappa:[0,1] \rightarrow[0,1]$ be a distortion function, $\phi=\left\{\varphi_{n}\right\}_{n \geq 1}$ a $\phi$-sequence, and $h:[a, b] \times \mathbb{R} \rightarrow \mathbb{R}$. If the composition operator $H$, associated with $h$, maps $\kappa \mathrm{BV}_{\phi}[a, b]$ in itself and satisfies the inequality

$$
\begin{array}{r}
\|H(u)-H(v)\|_{\kappa \phi} \leq \gamma\left(\|u-v\|_{\kappa \phi}\right), \\
\left(u, v \in \kappa \mathrm{BV}_{\phi}[a, b]\right),
\end{array}
$$

for any function $\gamma:[0, \infty) \rightarrow[0, \infty)$, verifying $\gamma(t) \rightarrow$ $\gamma(0)=0$, when $t \downarrow 0$, then there exists $\alpha, \beta \in \kappa \mathrm{BV}_{\phi}^{-}[a, b]$, such that

$$
h^{-}(t, x)=\alpha(t) x+\beta(t), \quad(t \in[a, b], x \in \mathbb{R}) .
$$

Proof. Let us fix $x, y \in \mathbb{R}, x \neq y$, and define $u(t):=x, v(t):=$ $y, t \in[a, b]$. Then $u, v \in \kappa \mathrm{BV}_{\phi}[a, b]$ and $\|u-v\|_{\kappa \phi}=|x-y|$. Then from inequality (90) and Lemma 16, we have

$$
\begin{gathered}
\varphi_{1}\left(\frac{|h(t, x)-h(t, y)-h(a, x)+h(a, y)|}{\gamma(|x-y|)}\right) \\
\quad \leq \kappa\left(\frac{t-a}{b-a}\right)+\kappa\left(\frac{b-t}{b-a}\right), \quad(t \in(a, b]) .
\end{gathered}
$$

And therefore

$$
\begin{aligned}
& |h(t, x)-h(t, y)-h(a, x)+h(a, y)| \\
& \leq \varphi_{1}^{-1}(2) \gamma(|x-y|) .
\end{aligned}
$$

Proceeding as in the proof of Theorem 22 we have that there is left regularization of $h^{-}(\cdot, x)$, for each $x \in \mathbb{R}$ and if $a \leq$ $r<a_{1}<b_{1}<a_{2}<\cdots<a_{m}<b_{m}=s \leq b, x, y \in \mathbb{R}, x \neq y$ we defined the zig-zag continuous functions $u, v:[a, b] \rightarrow \mathbb{R}$, as show below

$$
\begin{aligned}
& u(t)= \begin{cases}x, & a \leq t \leq a_{1} \text { or } t=a_{i}, i=1, \ldots, m \\
y, & b_{m} \leq t \leq b \text { or } t=b_{i}, i=1, \ldots, m \\
\text { affine, } & \text { other case, }\end{cases} \\
& u(t)= \begin{cases}y, & a \leq t \leq a_{1} \text { or } t=a_{i}, i=1, \ldots, m \\
x, & b_{m} \leq t \leq b \text { or } t=b_{i}, i=1, \ldots, m \\
\text { affine, } & \text { other case, }\end{cases}
\end{aligned}
$$

then $u, v \in \kappa \mathrm{BV}_{\phi}[a, b],(u-v)(t)=x-y, t \in[a, b]$ and for $s \in(a, b]$

$$
\begin{gathered}
\varphi_{m}\left(\frac{2\left|h^{-}(s, x)-h^{-}(s, y)\right|}{\gamma(|x-y|)}\right) \\
\leq \sum_{n=1}^{m} \varphi_{n}\left(\frac{2\left|h^{-}(s, x)-h^{-}(s, y)\right|}{\gamma(|x-y|)}\right) \\
\leq \kappa\left(\frac{s-a}{b-a}\right)+\kappa\left(\frac{b-s}{b-a}\right) \leq 2 .
\end{gathered}
$$

Hence,

$$
\begin{array}{r}
\left|h^{-}(s, x)-h^{-}(s, y)\right| \leq \frac{\varphi_{m}^{-1}(2)}{2} \gamma(|x-y|), \\
(s \in(a, b]) .
\end{array}
$$

And so $h^{-}(\cdot, x)$ is continuous in $(a, b]$. Now the result is a consequence of Theorem 22.

Corollary 24. Let $\kappa:[0,1] \rightarrow[0,1]$ be a distortion function, $\phi=\left\{\varphi_{n}\right\}_{n \geq 1}$ a $\phi$-sequence, $h:[a, b] \times \mathbb{R} \rightarrow \mathbb{R}$, and $H$ the composition operator associated with $h$. Suppose that $H$ maps $\kappa \mathrm{BV}_{\phi}[a, b]$ into itself and is uniformly continuous, then there exist $\alpha, \beta \in \kappa \mathrm{BV}_{\phi}^{-}[a, b]$, such that

$$
h^{-}(t, x)=\alpha(t) x+\beta(t), \quad(t \in[a, b], x \in \mathbb{R}),
$$

where $h^{-}(\cdot, x)$ is the left regularization of $h(\cdot, x)$ for all $x \in \mathbb{R}$.

Proof. Consider the modulus of continuity associated with $H$; that is,

$$
\begin{array}{r}
\gamma(t):=\sup \left\{\|H(u)-H(v)\|_{\kappa \phi}:\|u-v\|_{\kappa \phi}\right. \\
\left.\leq t, u, v \in \kappa \mathrm{BV}_{\phi}[a, b]\right\}, \\
(t \geq 0) .
\end{array}
$$


Then $\gamma(t) \geq 0, \gamma(0)=0$. Furthermore, if $t>0$ and $\|u-v\|_{\kappa \phi} \leq t$, we obtain

$$
\|H(u)-H(v)\|_{\kappa \phi} \leq \gamma(t) .
$$

Particularly, in the case where $t=\|u-v\|_{\kappa \phi}$, we have

$$
\begin{array}{r}
\|H(u)-H(v)\|_{\kappa \phi} \leq \gamma\left(\|u-v\|_{\kappa v}\right), \\
\left(u, v \in \kappa \mathrm{BV}_{\phi}[a, b]\right) .
\end{array}
$$

From Corollary 23 we obtain the conclusion.

Matkowski [27] introduced the notion of a uniformly bounded operator and proved that the generator of any uniformly bounded composition operator acting between general Lipschitz function normed spaces must be affine with respect to the function variable.

Definition 25. Let $\mathbb{Y}$ and $\mathbb{Z}$ be two metric (or normed) spaces. We say that a mapping $F: \mathbb{Y} \rightarrow \mathbb{Z}$ is uniformly bounded, if for any $t>0$, there exists a nonnegative real number $\gamma(t)$ such that for any nonempty set $B \subset \mathbb{Y}$ we have

$$
\operatorname{diam} B \leq t \Longrightarrow \operatorname{diam} F(B) \leq \gamma(t) .
$$

Remark 26. Every uniformly continuous operator or Lipschitzian operator is uniformly bounded.

Corollary 27. Let $\kappa:[0,1] \rightarrow[0,1]$ be a distortion function, $\phi=\left\{\varphi_{n}\right\}_{n \geq 1}$ a $\phi$-sequence, and $h:[a, b] \times \mathbb{R} \rightarrow \mathbb{R}$, such that function $h(t, \cdot): \mathbb{R} \rightarrow \mathbb{R}$ is continuous in respect to the second variable, for each $t \in(a, b]$. If the composition operator $H$, associated with $h$, maps $\kappa \mathrm{BV}_{\phi}[a, b]$ in itself and is uniformly bounded, then exists $\alpha, \beta \in \kappa \mathrm{BV}_{\phi}^{-}[a, b]$, such that

$$
h^{-}(t, x)=\alpha(t) x+\beta(t), \quad(t \in[a, b], x \in \mathbb{R}) .
$$

Proof. Take any $t>0$ and $u, v \in \kappa \mathrm{BV}_{\phi}[a, b]$ such that $\|u-v\|_{\kappa \phi} \leq t$. Since diam $\{u, v\} \leq t$, by uniform boundedness of $H$, we have diam $H(\{u, v\}) \leq \gamma(t)$; that is,

$$
\|H(u)-H(v)\|_{\kappa \phi}=\operatorname{diam} H(\{u, v\}) \leq \gamma\left(\|u-v\|_{\kappa \phi}\right) .
$$

From Theorem 22 we have (102).

\section{Acknowledgment}

This research has been party supported by the Central Bank of Venezuela. The authors want to thank the library staff of $\mathrm{BCV}$ for compiling the references.

\section{References}

[1] J. Appell, N. Guanda, and M. Väth, "Function spaces with the Matkowski property and degeneracy phenomena for composition operators," Fixed Point Theory, vol. 12, no. 2, pp. 265-284, 2011.

[2] J. Appell, Z. Jesús, and O. Mejía, "Some remarks on nonlinear composition operators in spaces of differentiable functions," Bollettino Della Unione Matematica Italiana, vol. 4, no. 3, pp. 321-336, 2011.
[3] A. A. Babaev, "On the structure of a certain nonlinear operator and itsapplication," Uchen. Zap. Azerbajdzh. Gos. Univ, vol. 4, pp. 13-16, 1961 (Russian).

[4] K. S. Mukhtarov, "On the properties of theoperator $F_{u}=$ $f(u(x))$ in the space $H_{\varphi}$," Sbornik Nauchm. Rabot Mat. Kaf. Dagestan Univ, pp. 145-150, 1967 (Russian).

[5] M. Josephy, "Composing functions of bounded variation," Proceedings of the American Mathematical Society, vol. 83, no. 2, pp. 354-356, 1981.

[6] J. Ciemnoczołowski and W. Orlicz, "Composing functions of bounded $\phi$-variation," Proceedings of the American Mathematical Society, vol. 96, no. 3, pp. 431-436, 1986.

[7] M. Chaika and D. Waterman, "On the invariance of certain classes of functions under composition," Proceedings of the American Mathematical Society, vol. 43, pp. 345-348, 1974.

[8] N. Merentes, "On the composition operator in $A C[a, b]$," Collectanea Mathematica, vol. 42, no. 3, pp. 231-238, 1991.

[9] N. Merentes, "On the composition operator in $R V_{\varphi}[a, b]$," Collectanea Mathematica, vol. 46, no. 3, pp. 231-238, 1995.

[10] N. Merentes and S. Rivas, "On the composition operator between $R V_{p}[a, b]$ and $B V[a, b]$," Studia Mathematica, vol. 1 , no. 3, pp. 237-292, 1998.

[11] P. B. Pierce and D. Waterman, "On the invariance of classes $\phi B V, \Lambda B V$ under composition," Proceedings of the American Mathematical Society, vol. 132, no. 3, pp. 755-760, 2004.

[12] J. Appell and N. Merentes, "Composing functions of bounded Koremblum variation," Dynamic Systems and Applications, vol. 22, pp. 197-206, 2013.

[13] W. Aziz, N. Merentes, and J. L. Sánchez, "A note on the composition of regularfunctions," Zeitschrift für Analysis und ihre Anwendungen. In press.

[14] J. Matkowski, "Functional equations and Nemytskiĭ operators," Fako de l'Funkcialaj Ekvacioj Japana Matematika Societo, vol. 25, no. 2, pp. 127-132, 1982.

[15] J. Appell, N. Guanda, N. Merentes, and J. L. Sánchez, "Some boundedness and continuity properties of nonlinear composition operators: a survey," Communications on Pure and Applied Analysis, vol. 15, no. 2-4, pp. 153-182, 2011.

[16] J. Matkowski and J. Miś, "On a characterization of Lipschitzian operators of substitution in the space $B V[a, b]$," Mathematische Nachrichten, vol. 117, pp. 155-159, 1984.

[17] C. Jordan, "Sur la série de Fourier," Comptes Rendus De L'Académie Des Sciences, pp. 228-230, 1881.

[18] J. Appell, J. Banas, and N. Merentes, Bounded variation and around. Gruyter. Series in nonlinear analysis and application.

[19] M. Avdispahić, "Concepts of generalized bounded variation and the theory of Fourier series," International Journal of Mathematics and Mathematical Sciences, vol. 9, no. 2, pp. 223244, 1986.

[20] N. Merentes and S. Rivas, El Operador De Composición En Espacios Con Algún Tipo De Variación Acotada, IX Escuela Venezolana de Matemáticas, Mérida, Venezuela, 1996.

[21] B. Korenblum, "An extension of the Nevanlinna theory," Acta Mathematica, vol. 135, no. 3-4, pp. 187-219, 1975.

[22] D. S. Cyphert and J. A. Kelingos, "The decomposition of functions of bounded $\kappa$-variation into differences of $\kappa$-decreasing functions," Polska Akademia Nauk, vol. 81, no. 2, pp. 185-195, 1985.

[23] M. Schramm, "Functions of $\Phi$-bounded variation and Riemann-Stieltjes integration," Transactions of the American Mathematical Society, vol. 287, no. 1, pp. 49-63, 1985. 
[24] S. K. Kim and J. Kim, "Functions of $\kappa \phi$-bounded variation," Bulletin of the Korean Mathematical Society, vol. 23, no. 2, pp. 171-175, 1986.

[25] M. Castillo, M. Sanoja, and I. Zea, “The spaces of functions of bounded $\kappa$-variation in the sense ofRiesz-Koremblum," Journal of Mathematical Control Science and Applications. In press.

[26] M. Castillo, S. Rivas, M. Sanoja, and I. Zea, "functions of bounded $K_{\varphi}$-variation in the sense of Riesz-Korenblum," Journal of Function Spaces and Applications, vol. 2013, Article ID 718507, 12 pages, 2013.

[27] J. Matkowski, "Uniformly bounded composition operators between general Lipschitz function normed spaces," Topological Methods in Nonlinear Analysis, vol. 38, no. 2, pp. 395-406, 2011.

[28] M. Kuczma, An Introduction to the Theory of Functional Equations and Inequalities, Polish Scientific Editors and Silesian University, Warszawa, Poland, 1885. 


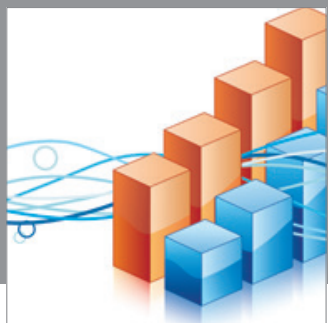

Advances in

Operations Research

mansans

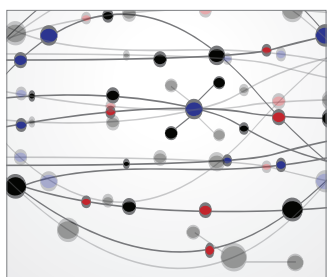

The Scientific World Journal
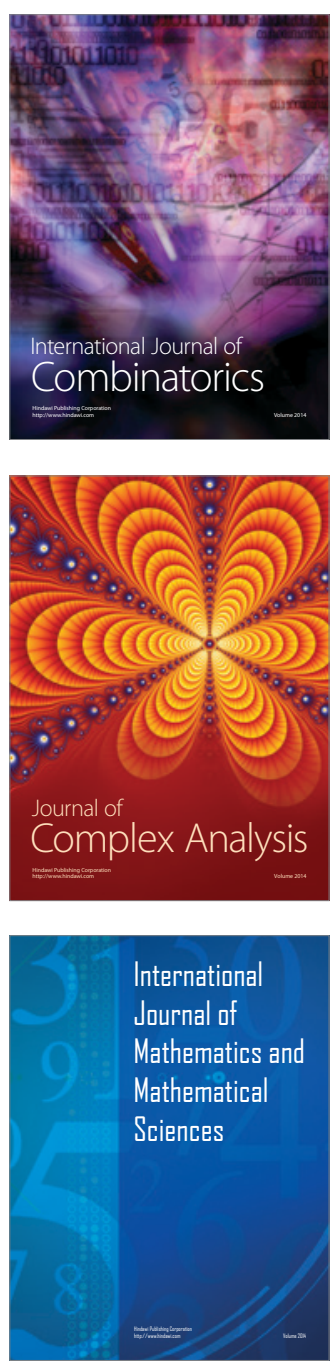
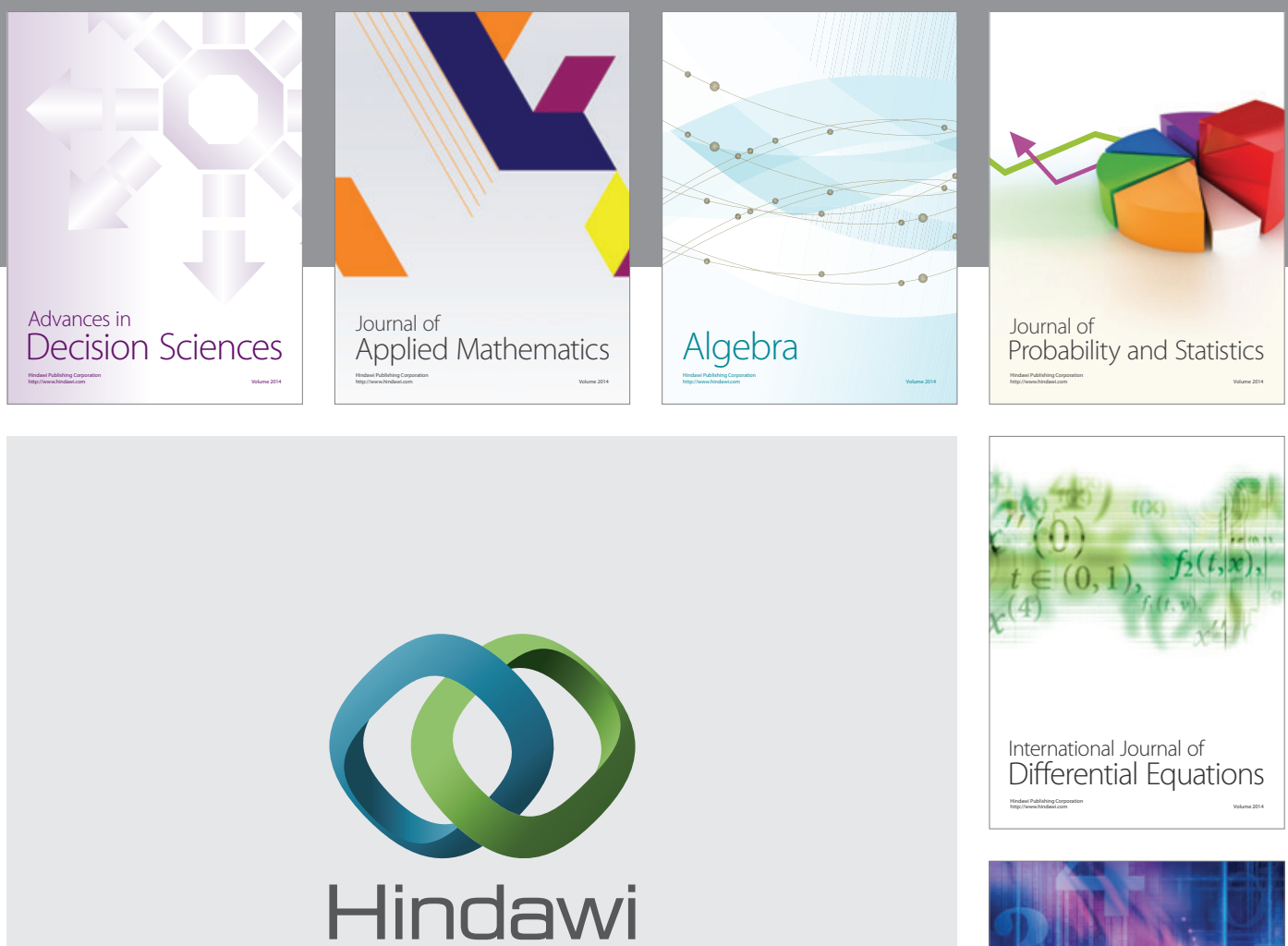

Submit your manuscripts at http://www.hindawi.com
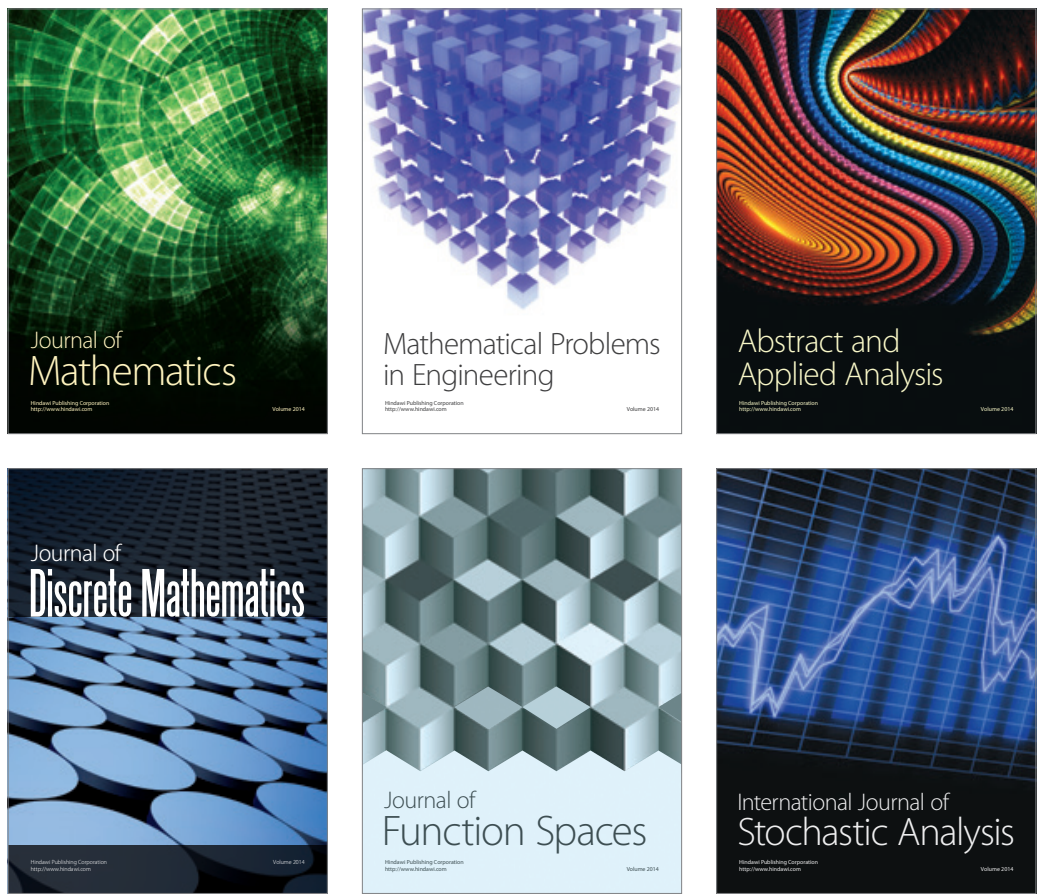

Journal of

Function Spaces

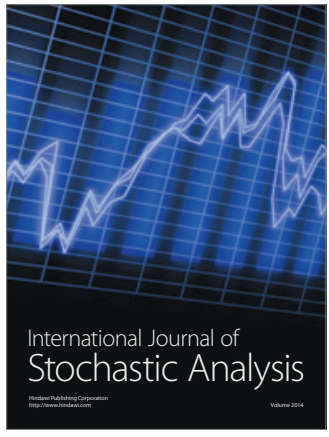

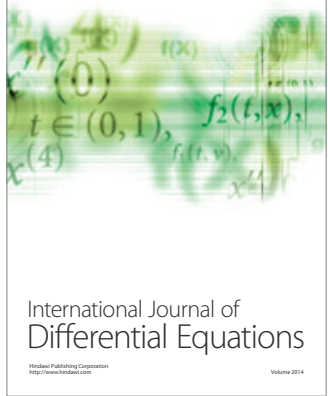
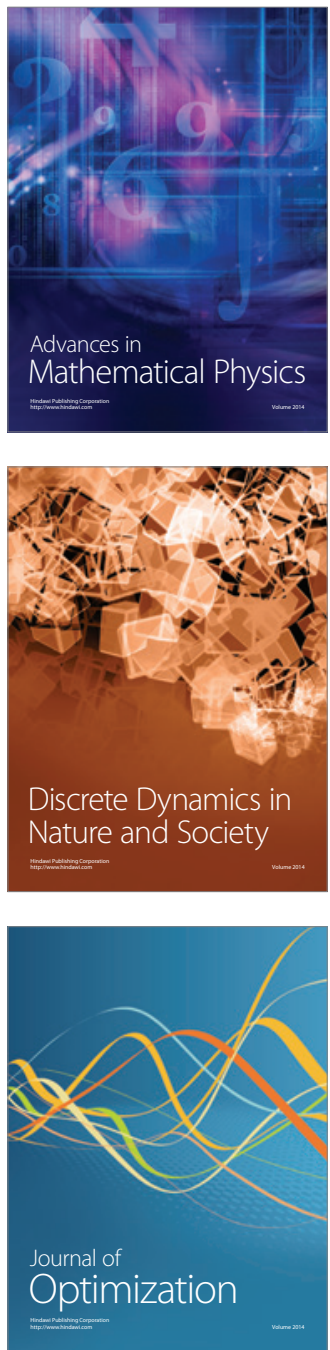